\title{
Development of an Automatic Sampling Module to Monitor Concentration of Liquid-Borne Nanoparticles
}

\author{
Yu-Shan Yeh, ${ }^{*}$ Kaoru Kondo, ${ }^{1}$ and Han-Fu Weng \\ Center for Measurement Standards, Industrial Technology Research Institute, \\ No. 321, Sec. 2, Kuang Fu Rd., Hsinchu 30011, Taiwan \\ ${ }^{1}$ RION Co., Ltd., 3-20-41 Higashimotomachi, Kokubunji, Tokyo 185-8533, Japan \\ (Received February 7, 2015; accepted January 8, 2016)
}

Keywords: dilution, automatic, concentration, nanoparticle

Particle detection in liquid reagents for various manufacturing processes is important, especially for the semiconductor industry, to maintain the yield and quality of the end products. As semiconductor devices become smaller, the critical diameters of particle contaminants also become smaller. A measurement system should be correctly calibrated to provide reliable measurements with suitable traceability. Owing to the lack of suitable standard solutions for particle concentration in terms of number, an automatic sampling module compliant with SEMI C-77 has been developed to generate standard solutions with particle concentration as low as $10^{2}-10^{3}$ particles $/ \mathrm{cm}^{3}$ depending on the quality of the ultrapure water (UPW) used. With the precise control of flow rates at low and high flux ranges, a dilution factor as high as $4.8 \times 10^{9}$ can be achieved with high accuracy. A concentration standard of polystyrene latex (PSL) nanoparticles with a size of $>30 \mathrm{~nm}$ was used as stock solution to verify the particle numbers counted by light scattering. The homogeneity of the dilution process was evaluated on the basis of the variation in particle counts during $10 \mathrm{~min}$ after sample injection. This automatic system can be applied to the real-time monitoring of the nanoparticles in liquid reagents used in device fabrication.

\section{Introduction}

Particle control is crucial for the semiconductor industry and the upstream companies providing raw materials for device fabrication. A liquid particle counter (LPC) is widely used to monitor particle-number concentrations in manufacturing processes because of its capability of fast and reliable detection and its high throughput. To avoid the error from coincidence loss in particle detection, the particle concentration should be kept below the specified upper limit of the LPC, which varies between $10^{3}-10^{5}$ particles $/ \mathrm{mL}$ depending on the instrument design and the background values. Since it is not easy to carry out a manual dilution with sufficient accuracy and repeatability in this concentration range, an automatic sampling module is necessary to dilute the sample solution to a suitable level and produce a sample solution that is appropriate for concentration measurements with the LPC. In this paper, an automatic sampling module compliant with SEMI C- $77^{(1,2)}$ is described, the performance of which is verified using various parameters including the stability of flow rates, linearity over the dilution range, and the homogeneity of the concentration after dilution.

*Corresponding author: e-mail: yushan@itri.org.tw 


\section{Materials and Methods}

\subsection{Automatic sampling module and particle sensor}

The automatic sampling module XL-21 (Fig. 1) was manufactured by RION, Japan, with a specified dilution factor of 4000-22000. To verify its performance over a particle-size range from 30 to $300 \mathrm{~nm}$, two types of sensors, KS-19F and KS-42B (RION, Japan), were used. The detection efficiencies at 30 and $300 \mathrm{~nm}$ were 0.01 and $100 \%$, respectively. Each time before a sample was injected, the entire system was flushed with ultrapure water (UPW, $18 \mathrm{M} \Omega$ ) for at least $2 \mathrm{~h}$ until no significant background signal was detected. When switching from UPW to a sample, the syringe was purged at least 20 times to replace any residual water with the sample solution. The flow rate in the particle sensor was fixed at $10 \mathrm{~mL} / \mathrm{min}$, and the sampling time was set to 1 min per data point.

\subsection{Verification of flow rates}

The principle of the design in the sampling module is illustrated in Fig. 2. Following the suggestion in SEMI C-77, the automated dilution process was carried out by mixing the sample with UPW, where the dilution factor was determined from the flow rate of UPW $\left(U_{0}\right)$ and the injection flow rate of a syringe pump $\left(U_{1}\right)$. Since $U_{0}$ could be altered by adjusting the sample flow rate of the particle sensor $\left(U_{2}\right)$ and that of the bypass flow $\left(U_{3}\right)$, the dilution factor for the automated dilution $\left(D F_{\mathrm{a}}\right)$ was calculated as

$$
D F_{\mathrm{a}}=\frac{U_{1}}{U_{0}+U_{1}}=\frac{U_{1}}{U_{2}+U_{3}} .
$$

The flow rates of $U_{2}$ and $U_{3}$ were verified by weighing the mass of water in a certain time period recorded using a stopwatch. In our tests, the length of the time period was about $1 \mathrm{~min}$. The flow rate of $U_{1}$ was also verified by weighing the first time the syringe pump was installed.

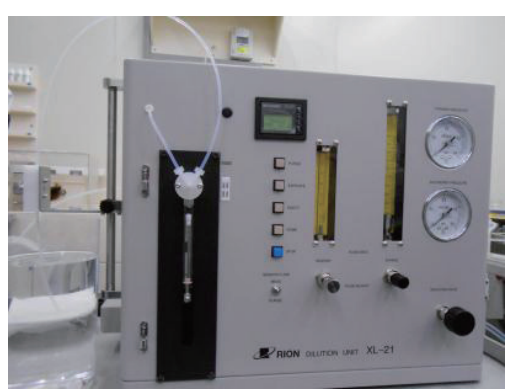

Fig. 1. (Color online) Automatic sampling module RION XL-21.

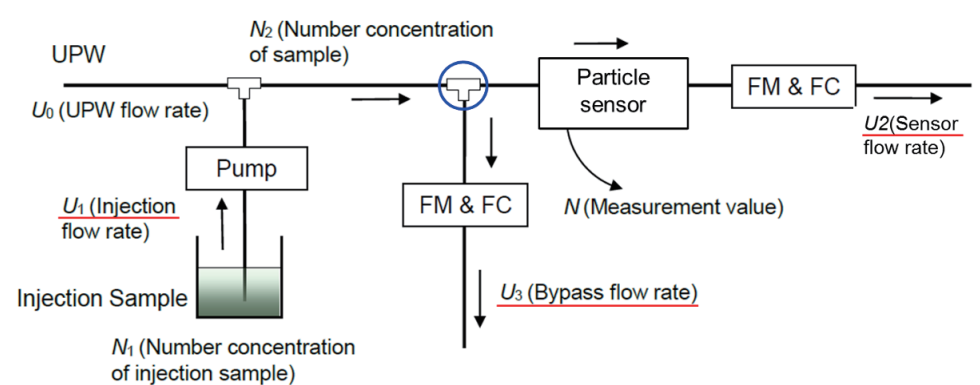

Fig. 2. (Color online) Schematic view of the sampling module (revised from Fig. 1 of SEMI C-77). 


\subsection{Preparation of injected sample}

Monodispersed, commercially available polystyrene latex (PSL) nanoparticles with certified concentrations were used as particle standards. The JSR SC-032-S of $309 \mathrm{~nm}$ size and Thermo $3030 \mathrm{~A}$ of $30 \mathrm{~nm}$ size were selected to cover the size range from 30 to $300 \mathrm{~nm}$. To prepare the injection sample as shown in Fig. 1, $5 \mu \mathrm{L}$ of stock solution $\left(V_{\mathrm{PSL}}\right)$ was carefully pipetted into a glass beaker and $\sim 1000 \mathrm{~mL}$ of UPW $\left(V_{\mathrm{UPW}}\right)$ was added to achieve a dilution factor of $\sim 2 \times 10^{5}$. The dilution factor for the manual dilution $\left(D F_{\mathrm{m}}\right)$ is calculated as

$$
D F_{\mathrm{m}}=\frac{V_{\mathrm{PSL}}}{V_{\mathrm{PSL}}+V_{\mathrm{UPW}}}
$$

\section{Results and Discussion}

The performance of the sampling module was first verified with 309-nm-diameter PSL nanoparticles and the particle sensor KS-42B for which a full detection scheme was adopted. Then the performance for particles $<100 \mathrm{~nm}$ in diameter was tested with 30-nm-diameter PSL nanoparticles and the particle sensor KS-19F. The test results are described and discussed separately in the following subsections.

\subsection{Verification of test conditions}

Bypass and sensor flow rates were regulated by the flow controller and pressure gauges (as shown in Fig. 1). To verify the stability of the flow rates, the flow rates were measured 3 times before the experiments and checked again after $6 \mathrm{~h}$ of experiments. The results are summarized in Table 1. Both flow rates remained the same with a variation $<0.5 \%$.

In real tests, the particle sensor started to collect signals when the sample was injected. Owing to the void volume of the tubing, it took some time for the PSL nanoparticles to reach the detector. To check the time required for the particles to travel, the sampling time was set to $10 \mathrm{~s}$ to monitor the rise time of the signals. As shown in Fig. 3(a), the particle counts kept increasing at the beginning and reached a stable level after $40 \mathrm{~s}$. Therefore, it should be noted that when the sampling time was set at 1 min per data point, as in the normal measurements, all the data analyses

Table 1

Stability test of the bypass and sensor flow rates.

\begin{tabular}{lcc}
\hline & Bypass flow rate $(\mathrm{mL} / \mathrm{min})$ & Sensor flow rate $(\mathrm{mL} / \mathrm{min})$ \\
\hline Trial 1 & 1099.47 & 9.97 \\
Trial 2 & 1098.80 & 9.95 \\
Trial 3 & 1098.69 & 9.98 \\
Average & 1098.99 & 9.97 \\
SD & 0.422 & 0.017 \\
After 6 h & 1099.81 & 9.93 \\
Variation (\%) & 0.07 & -0.42 \\
\hline
\end{tabular}




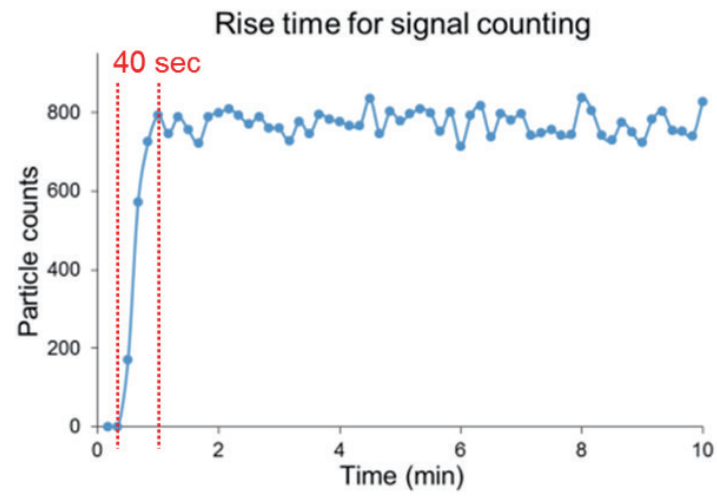

(a)

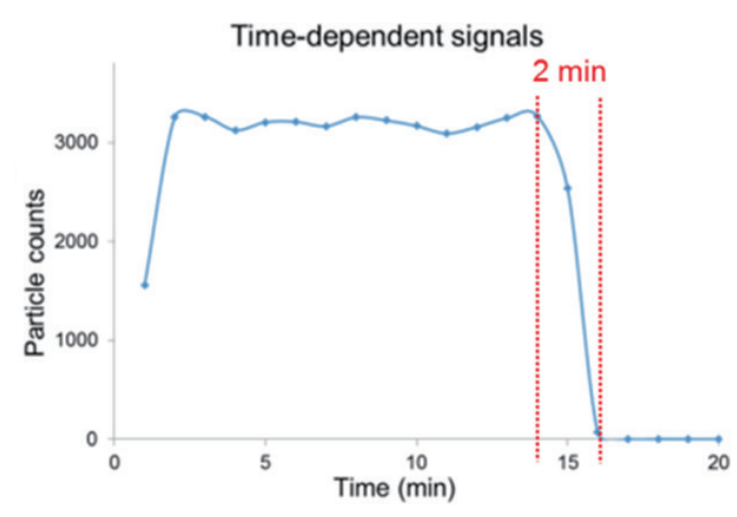

(b)

Fig. 3. (Color online) Rise (a) and fall (b) times of particle counts after sample injection and after the sample injection had been stopped.

were carried out with data points starting from the second one. In addition to the rise time of the signal, the fall time of the signal was also monitored. As shown in Fig. 3(b), it took at least 2 min for the particle counts to fall into the background level and for the device to be ready for the next injection.

\subsection{Linearity test for 309-nm-diameter PSL nanoparticles}

The linearity of the particle counts over the entire dilution range was verified with JSR SC032-S $(309 \mathrm{~nm})$. By adjusting the bypass and sensor flow rates, the value of $D F_{\text {a }}$ could be varied from 4100 to 22200 (Table 2). Multiplied by the dilution factor in the manual preparation process $\left(D F_{\mathrm{m}}\right)$, the overall dilution factor could be high as $4.8 \times 10^{9}$. The number concentrations (particles/ $\mathrm{mL}$ ) were calculated by dividing the specified concentration of the stock solution by the dilution factors. Compared with the particle counts measured using the particle sensor, the linearity over the dilution range could be verified from the $R^{2}$ value of near unity obtained from linear regression analysis (Fig. 4). The intercept of the best fit line was forced to be zero owing to low background signals of the particle sensor. The slope $(0.961)$, which represents the average recovery of concentration using this sampling module, showed $<5 \%$ deviation from the specified value. The homogeneity of concentration after dilution could be verified using the coefficient of variation $(\mathrm{CV})$ from 5 data points, which was defined as

$$
C V(\%)=\frac{\text { Standard deviation }}{\text { Average }} \times 100 \%
$$

It was observed from the data in Table 2 that the $C V$ values at different sample concentrations were all $<5 \%$, which indicated that the output concentration after the automatic sampling module was homogeneous and stable with time. 
Table 2

Homogeneity test results for 309 and $30 \mathrm{~nm}$ PSL nanoparticles.

\begin{tabular}{|c|c|c|c|c|c|}
\hline \multicolumn{3}{|c|}{ JSR SC-032-S (309 nm) } & \multicolumn{3}{|c|}{ Thermo 3030A $(30 \mathrm{~nm})$} \\
\hline$D F_{\mathrm{a}}$ & $D F_{\mathrm{m}} \times D F_{\mathrm{a}}$ & $C V(\%)$ & $D F_{\mathrm{a}}$ & $D F_{\mathrm{m}} \times D F_{\mathrm{a}}$ & $C V(\%)$ \\
\hline 4100 & $8.26 \mathrm{E}+08$ & 3.7 & 4100 & $8.74 \mathrm{E}+08$ & 2.8 \\
\hline 5100 & $1.02 \mathrm{E}+09$ & 1.5 & 5100 & $1.11 \mathrm{E}+09$ & 4.4 \\
\hline 5857 & $1.18 \mathrm{E}+09$ & 4.6 & 5857 & $1.25 \mathrm{E}+09$ & 2.2 \\
\hline 6833 & $1.38 \mathrm{E}+09$ & 1.1 & 6833 & $1.46 \mathrm{E}+09$ & 2.1 \\
\hline 7625 & $1.60 \mathrm{E}+09$ & 1.2 & 7625 & $1.74 \mathrm{E}+09$ & 2.1 \\
\hline 8714 & $1.83 \mathrm{E}+09$ & 2 & 8714 & $1.98 \mathrm{E}+09$ & 2.3 \\
\hline 10200 & $2.04 \mathrm{E}+09$ & 1 & 10200 & $2.22 \mathrm{E}+09$ & 3.8 \\
\hline 11375 & $2.45 \mathrm{E}+09$ & 2.6 & 11375 & $2.61 \mathrm{E}+09$ & 3.4 \\
\hline 12200 & $2.57 \mathrm{E}+09$ & 1.7 & 13500 & $3.08 \mathrm{E}+09$ & 4.4 \\
\hline 13500 & $2.90 \mathrm{E}+09$ & 0.8 & 15857 & $3.71 \mathrm{E}+09$ & 6.9 \\
\hline 14429 & $3.15 \mathrm{E}+09$ & 1 & 18200 & $4.17 \mathrm{E}+09$ & 3.4 \\
\hline 15857 & $3.49 \mathrm{E}+09$ & 1.5 & 22200 & $4.90 \mathrm{E}+09$ & 4.1 \\
\hline 16833 & $3.67 \mathrm{E}+09$ & 1.6 & & & \\
\hline 18200 & $3.92 \mathrm{E}+09$ & 2 & & & \\
\hline 20200 & $4.41 \mathrm{E}+09$ & 1.1 & & & \\
\hline 22200 & $4.89 \mathrm{E}+09$ & 2.3 & & & \\
\hline
\end{tabular}



Fig. 4. (Color online) Linearity test for 309-nmdiameter PSL nanoparticles.

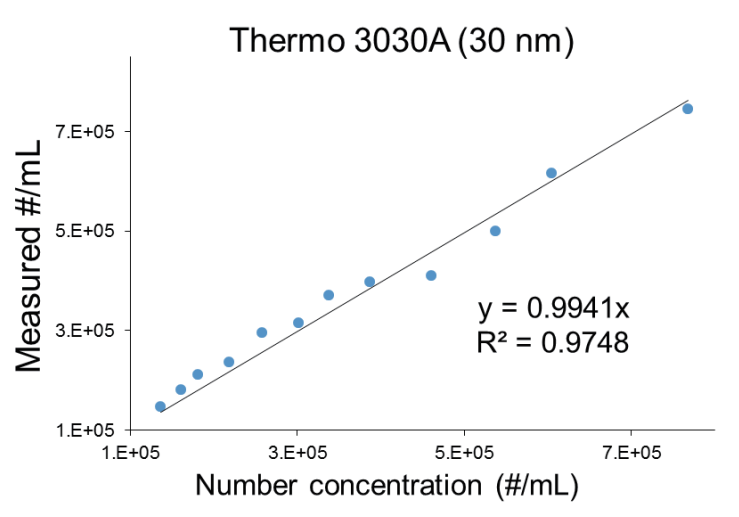

Fig. 5. (Color online) Linearity test for 30-nmdiameter PSL nanoparticles.

\subsection{Linearity test for 30-nm-diameter PSL nanoparticles}

The linearity of the sampling module for 30-nm-diameter PSL nanoparticles was verified in a process similar to that described in $\S 3.2$, except that some of the test conditions were skipped to save some time (Table 2). From the results of the linear regression analysis shown in Fig. 5, the $R^{2}$ value indicated a satisfactorily linear response between the sample concentration and the particle counts collected by the particle sensor over the entire dilution range. The slope (0.9941) indicated an almost full recovery of the stock concentration. The $C V$ values at different sample concentrations were also smaller than $5 \%$, except for one outlier. 


\section{Conclusions}

An automatic sampling module was successfully developed and validated for nanoparticles ranging from 30 to $300 \mathrm{~nm}$ in diameter. The linearity of the automated dilution process and the homogeneity of the final concentrations were verified over the entire dilution range. The output concentration showed less than $5 \%$ deviation from the specified value, indicating the qualifications of the module to generate concentration standards within its dilution range. This module could be a useful tool for the semiconductor industry to achieve real-time monitoring of the liquid-borne nanoparticles present in the production line.

\section{Acknowledgements}

This work was supported by Metal Industries Research \& Development Centre, Taiwan, and the Bureau of Standards, Metrology \& Inspection, M.O.E.A., Taiwan.

\section{References}

1 Japan Liquid Chemicals Committee: Test Method for Determining the Counting Efficiency of Liquid-Borne Particle Counters for which the Minimum Detectable Particle Size is between $30 \mathrm{~nm}$ and $100 \mathrm{~nm}$, SEMI Draft Document C-77 (2012).

2 K. Kondo, M. Shimmura, and T. Tabuchi: Proc. 13th Conf. INE (International Nano-Electrochemistry, 2013) pp. 47-55. 